\title{
Factors Affecting Quality of Life of the Homebound Elderly Hemiparetic Stroke Patients
}

\author{
Seitchi Takemasa ${ }^{1)^{*}}$, Ryoma Nakagoshi ${ }^{2)}$, Masahito Murakami ${ }^{1}$, Masayuki Uesugi ${ }^{1)}$, \\ Yuri Inoue ${ }^{1)}$, Makoto Gotou ${ }^{1)}$, Hideki Koeda ${ }^{1)}$, Susumu Naruse ${ }^{1)}$ \\ 1) Faculty of Rehabilitation, Kobe International University: 9-1-6 Koyou-cho Higashinada-ku, Kobe \\ City, Hyogo 658-0032, Japan \\ 2) Department of Rehabilitation, Geriatric Health Services Facility Elder Village, Japan
}

\begin{abstract}
Purpose] This study examined the quality of life (QOL) of homebound elderly hemiparetic stroke patients and factors that affect it. [Subjects] The subjects of the study were 21 homebound elderly hemiparetic stroke patients who were 65 years old or over and required care for daily living (12 males and 9 females, average age: $79.3 \pm$ 8.4 years old). Their physical and psychological conditions, QOL, and other characteristics were researched. [Methods] The Functional Independence Measure (FIM) was used for the activities of daily living (ADL) assessment, and the MOS 36-Item Short-Form Health Survey (SF-36, Japanese version 1.2) was used for the QOL assessment. [Results] No correlations were observed between the QOL of homebound elderly hemiparetic stroke patients and their age and gender. However, the results showed that their QOL was affected by their independence in ADL, bedridden degree, and care-need level. [Conclusion] These results suggest that in order to improve the QOL of homebound elderly hemiparetic stroke patients, ongoing rehabilitation to improve independence in ADL and lower the bedridden degree and care-need level is required.

Key words: QOL, Homebound elderly hemiparetic stroke patients, ADL
\end{abstract}

(This article was submitted Aug. 5, 2013, and was accepted Sep. 22, 2013)

\section{INTRODUCTION}

Japan is one of the world's fastest aging societies, and the increase in the elderly disabled requiring long-term care is becoming a social problem. According to the LongTerm Care Insurance Report 2012 issued by the Ministry of Health, Labour and Welfare (MHLW), 5,306,000 people, including the bedridden elderly, now have a Certification of Long-Term Care Need ${ }^{1)}$, and the figure is expected to continue to increase.

According to the MHLW Report of Basic Research on Japanese Life 2010, cerebrovascular diseases (strokes) are the most common cause for disabilities that require care, accounting for $21.5 \%$. The percentage increases as the care-need level increases. For people with the highest careneed, which is level $\mathrm{V}, 30.8 \%$ became elderly disabled due to strokes ${ }^{2)}$. Generally speaking, risk of stroke increases with age. Since Japan is rapidly aging, people suffering from strokes are expected to increase. Taking appropriate measures for the bedridden elderly is urgently required ${ }^{3}$. In addition, strokes often cause aftereffects. Under the government policy that reduces medical spending and encourages

*Corresponding author. Seiichi Takemasa (E-mail: takemasa@kobe-kiu.ac.jp)

(C2014 The Society of Physical Therapy Science. Published by IPEC Inc. This is an open-access article distributed under the terms of the Creative Commons Attribution Non-Commercial No Derivatives (by-ncnd) License $<$ http://creativecommons.org/licenses/by-nc-nd/3.0/>. elderly people to be treated at home, discharging them from institutions as soon as possible, services for homebound elderly hemiparetic stroke patients that consider their quality of life (QOL) are required for them to live securely and stably at home.

This study looked at homebound elderly hemiparetic stroke patients, researched their physical conditions and QOL, and analyzed and examined factors that affect the QOL to discover how these patients can maintain their QOL at home after being discharged from institutions.

\section{SUBJECTS AND METHODS}

A questionnaire was administered to 21 homebound elderly hemiparetic stroke patients who were 65 years old or over, used home-visit care services, did not suffer from obvious dementia, and were able to answer the questionnaire (12 males and 9 females, average age: $79.3 \pm 8.4$ years old). We visited their homes and researched their physical functions and QOL.

In term of ethical considerations, details of the study were explained to subjects verbally and in writing, and signed consent forms were received from subjects.

The research items were subject age, gender, daily life independence level of the elderly disabled persons (bedridden degree), care-need level, activities of daily living (ADL), and QOL.

The Functional Independence Measure (FIM) was used for the ADL assessment ${ }^{4}$. This measure consists of 13 mobility items and 5 communication and social cognition 
items. Subject ADL performance is assessed on a scale of 1 to 7. A full score is 126 points in total, meaning fully-independent, and the lowest possible score is 18 points, meaning total assistance is required.

The MOS 36-Item Short-Form Health Survey (SF-36, Japanese-version 1.2) was used for the QOL assessment ${ }^{5}$. This measure is a comprehensive health-related QOL scale, and is commonly used for assessment with subjective outcome measures. The subscales consist of 8 sections: Physical Functions (PF), Role Physical (RP), Body Pain (BP), General Health (GH), Vitality (VT), Social Functions (SF), Role Emotional (RE), and Mental Health (MH). There are 36 questions. The raw score for each subscale can be from 0 to 100 points. Higher scores indicate higher QOL. Nationwide standard scores for each gender and age are available, and can be easily compared with the scores of the subject group. This is an advantage of this measure.

To examine the correlations between the QOL and ADL and the burden felt by caregivers, Spearman's rank method was used. For comparisons between two groups, the MannWhitney U test was used. For statistical analysis, we used Stat Soft's statistical analysis software, STATISTICA. A significance level of 5\% was considered statistically significant.

\section{RESULTS}

For the ADL independence of the homebound elderly hemiparetic stroke patients, the subjects' total FIM scores ranged from 24 to 123 points (average: $72.7 \pm 34.1$ ), indicating that some required total assistance and some were nearly fully-independent. For the bedridden degree, only 1 subject $(4.8 \%)$ was nearly independent and able to go out alone. Eight subjects (38.1\%) were independent at home but required assistance for going out, and 12 subjects (57.1\%) required assistance both at home and when going out. Regarding their care-need levels, 2 subjects were at the assistance-need level, 5 subjects were at care-need level I, 3 subjects were at care-need level III, 6 subjects were at careneed level IV, and 5 subjects were at care-need level V.

The average QOL of the homebound elderly hemiparetic stroke patients was lower in six subscales, excluding $\mathrm{RP}$ and RE, and was particularly lower for the PF and MH subscales, than the deviation score of 50 of the nationwide standard scores for the same age group (Table 1).

No correlations were observed between the QOL and age and gender.

For the correlations between the QOL and total FIM scores of the homebound elderly hemiparetic stroke patients, the PF and VT subscales for QOL were significantly high when ADL independence was high. As for the correlations between the QOL and FIM items, the Physical Functions and Vitality significantly correlated with all mobility items. When ADL independence was high, the PF and VT subscales for QOL were also high $(\mathrm{r}=0.71-0.84, \mathrm{p}<0.001)$. The SF subscales were significantly correlated with the independence of transfer (from bed to chair, wheelchair to toilet seat, etc.) and movement (from a room/place to another room/place). When patients are more capable in transfer and
Table 1. Comparison between disabled elderly person's QOL and the national standard deviation value (50)

\begin{tabular}{lc}
\hline & Deviation value \\
\hline SF-36 & Mean \pm SD \\
\hline Physical Function & $29.3 \pm 11.4$ \\
Role Physical & $55.8 \pm 6.2$ \\
Body pain & $49.8 \pm 9.6$ \\
General Health & $47.9 \pm 13.8$ \\
Vitality & $45.8 \pm 10.8$ \\
Social Function & $47.1 \pm 14.5$ \\
Role Emotional & $52.0 \pm 7.8$ \\
Mental Health & $43.1 \pm 7.8$ \\
\hline
\end{tabular}

movement, their spheres of activity become broader and the SF scores also become higher. The BP subscale showed significant negative correlations with transfer $(-0.55, \mathrm{p}<0.05)$ and movement $(\mathrm{r}=-0.71, \mathrm{p}<0.01)$, suggesting that patients complain more about body pain when they are more capable in transfer and movement and their spheres of activity are broader (Table 2).

For the correlations between the QOL and bedridden degree, subjects with a higher bedridden degree showed significantly lower PF $(r=-0.74, p<0.01)$ and VT scores $(\mathrm{r}=-0.61, \mathrm{p}<0.05)$ and complained less about body pain $(\mathrm{r}=-0.55, \mathrm{p}<0.05)$. For the correlations between the QOL and care-need level, subjects with a higher care-need level showed significantly lower PF scores $(r=-0.50, \mathrm{p}<0.05)$ and complained less about body pain $(r=-0.55, \mathrm{p}<0.05)$ (Table 2).

\section{DISCUSSION}

Although the stroke mortality rate is decreasing in Japan, more and more people are suffering from strokes. Strokes often cause aftereffects, leaving patients with disabilities. Since shortening the duration of hospital/institution stays is encouraged today, services for homebound elderly hemiparetic stroke patients that consider their QOL are required for them to live securely and stably at home after discharge from hospitals or institutions. This study looked at homebound elderly hemiparetic stroke patients and their QOL, and analyzed and examined factors that affect QOL. The results indicated that patient age or gender does not directly affect QOL. The results, however, showed that their QOL was affected by the bedridden degree, care-need level, and ADL independence.

The results showed that the QOL of the homebound elderly hemiparetic stroke patients was lower in six subscales, excluding RP and RE, than the deviation score of the same age group. Their physical activity and spheres of activity are limited, and they have less social interaction. They lead their daily lives within the bounds of their abilities. Our results suggested that homebound elderly hemiparetic stroke patients are more anxious about their future health and are depressed.

As for the correlations between the ADL and QOL of 
Table 2. Correlation coefficient of disabled elderly person's QOL (SF-36) and independence in ADL, bedridden degree, and careneed level

\begin{tabular}{|c|c|c|c|c|c|c|c|c|c|c|c|c|}
\hline \multicolumn{13}{|c|}{ SF-36 } \\
\hline ADL and others & & $\mathrm{PH}$ & & $\mathrm{RP}$ & $\mathrm{B}$ & & GH & $\mathrm{V}^{\prime}$ & & SF & $\mathrm{RE}$ & $\mathrm{MH}$ \\
\hline \multirow{7}{*}{ FIM } & Self-care & 0.77 & $* *$ & -0.50 & -0.47 & & 0.02 & 0.61 & $* *$ & 0.25 & 0.08 & 0.35 \\
\hline & Excretion control & 0.71 & $* *$ & -0.49 & -0.42 & & 0.17 & 0.52 & * & 0.29 & 0.05 & 0.34 \\
\hline & Transfer & 0.78 & $* *$ & -0.42 & -0.55 & * & -0.05 & 0.51 & * & $0.51 *$ & -0.19 & 0.08 \\
\hline & Locomotion & 0.84 & $* *$ & -0.50 & -0.71 & $* *$ & -0.01 & 0.55 & $*$ & $0.52 *$ & -0.29 & 0.11 \\
\hline & Communication & 0.17 & & -0.16 & -0.22 & & -0.13 & 0.21 & & 0.10 & -0.16 & -0.10 \\
\hline & Social cognition & 0.30 & & -0.19 & -0.25 & & -0.26 & 0.30 & & 0.46 & -0.05 & 0.05 \\
\hline & & 0.78 & $* *$ & -0.46 & -0.53 & * & -0.05 & 0.53 & $*$ & 0.47 & -0.01 & 0.29 \\
\hline Bedridden level & & -0.74 & $* *$ & 0.45 & 0.61 & * & 0.21 & -0.61 & * & -0.26 & 0.13 & -0.20 \\
\hline Care-need level & & -0.50 & & 0.20 & 0.55 & * & 0.31 & -0.10 & & -0.37 & 0.43 & 0.08 \\
\hline
\end{tabular}

$* * \mathrm{p}<0.01, * \mathrm{p}<0.05$

PF: Physical function, RP: Role physical, BP: Body pain, GH: General health, VT: Vitality, SF: Social function, RE: Role emotional, MH: Mental health

ordinary elderly people, Sugisawa ${ }^{6)}$ reported that their QOL correlates with their ADL independence. Fujita ${ }^{7}$ also reported that physical ADL strongly correlate with QOL. Sasuga $^{8)}$ noted that extended ADL (8 ADL items and 12 IADL items) are a factor that affects the QOL of the homebound disabled. Our results also indicated that the ADL independence of homebound elderly hemiparetic stroke patients correlates with their QOL, such as their Physical Functions and Vitality, verifying the results of the above-mentioned researchers. The QOL of homebound elderly hemiparetic stroke patients also significantly correlated with the bedridden degree and care-need level, which indicate mobility and amount of assistance required. When patient bedridden degree and care-need level were higher, the QOL was lower. Therefore, our results suggested that improving the ADL independence and lowering the bedridden degree and careneed level are required to improve the QOL of homebound elderly hemiparetic stroke patients.

Regarding the correlations between the QOL and FIM items, the PF and VT subscales were significantly correlated with independence in the mobility items. When transfer and movement ability are higher, individuals have broader spheres of activity and higher social functions. Therefore, in order to improve the QOL, such as physical functions, vitality and social functions, of people requiring long-term care, improving ADL independence is, of course, important. It is, however, particularly important to assure their movement ability, as Fukuya ${ }^{9)}$ has noted. Patients with higher transfer and movement ability also tended to have broader spheres of activity and to complain more about body pain. With the development or worsening of such body pain, their movement ability may be restricted, obstructing their social and daily activity. This contradicts the improved social functions with broader spheres of activity. When patients have more opportunities to engage in activity, the physical bur- den imposed on their bodies increases, and they have more risk of suffering from body pain. Therefore, simply improving their physical functions and ability is insufficient. The risk of developing new body pain must be assessed and addressed appropriately in order to improve QOL, particularly the individual's social functions and body pain.

The results of this study suggested that, in order to improve the QOL of homebound elderly hemiparetic stroke patients, continuous rehabilitation to maintain and improve patient ADL ability and assess and relieve the pain that patients feel when they move their bodies is required.

\section{REFERENCES}

1) Ministry of Health, Labour and Welfare: Nursing insurance business report. http://www.mhlw.go.jp/topics/kaigo/osirase/jigyo/11/index.html (Accessed May 18, 2013)

2) Ministry of Health, General condition of national life base investigation in 2010. http://www.mhlw.go.jp/toukei/saikin/hw/k-kyosa/k-kyosa10/4-3. html (Accessed May 18, 2013)

3) Iwamoto $T$, Kiuchi A: Cerebrovascular diseases in the elderly from gerontological aspects. Nippon Ronen Igakkai Zasshi, 2003, 40: 476-479. [Medline] [CrossRef]

4) Chino N, Sonoda S, Ryu M, et al.: Function evaluation of stroke patients - Actually application of SAIAS and FIM- Tokyo: Shupringer-Verlag, 1997, pp 42-96.

5) Fukuhara S, Suzukamo Y: Manual of SF-36v2 Japanese version Institute for Health Outcome \& Process Evaluation Research, Kyoto, 2004

6) Sugisawa H: Difference in impact of social support on morale and medical utilization between elderly with low activity of daily living and elderly with high activity of daily living. Nippon Koshu Eisei Zasshi, 1993, 40: 171-180. [Medline]

7) Fujita T, Ohtuka T, Taniguchi K: Subjective well-being of the Japanese elderly and its correlates. Soc Gerontol, 1989, 29: 75-85.

8) Sasuga Y: The influential factors to the quality of life of elderly persons a home with disabilities. J of Japan Academy of Home Health Care, 2001, 4: 32-39.

9) Fukuya Y: Geriatric rehabilitation - The role of physical therapist in com—munity based rehabilitation. Rigaku Ryou Hogaku, 1990, 17: 301302 . 